\title{
BUILT UP CHANGE DETECTION BASED ON STRUCTURAL CLASSIFICATION OF DIGITAL ELEVATION MODELS
}

\author{
Sh. Hosseini ${ }^{1}$, F. Tabib Mahmoudi ${ }^{1 *}$, A. Aboutalebi ${ }^{2}$ \\ ${ }^{1}$ Dept. of Geomatic Engineering, Civil Engineering Faculty, Shahid Rajaee Teacher Training University, Tehran, Iran - \\ shararehhosseini@yahoo.com,fmahmoudi@sru.ac.ir \\ ${ }^{2}$ National Cartographic Center, Tehran, Iran - abootalebi1349@gmail.com
}

Commission VI, WG VI/4

KEY WORDS: Buildings, Normalized DSM, Segmentation, Structural Classification, Change Detection

\begin{abstract}
:
Multi temporal changes in built up areas are mainly caused by natural disasters (such as floods and earthquakes) or urban sprawl. Detecting these changes which consist of construction, destruction and renovation of buildings can play an important role in updating three dimensional city models and making the right decisions for urban management. Generally, change detection methods based on multi temporal remotely sensed data can be divided into 2D and 3D categories. Three dimensional change detection methods are suitable for identifying the changes of three dimensional objects such as buildings and their results are more close to reality. The objective of this study is to provide an effective method for 3D change detection of buildings in urban areas based on Digital Elevation Models (DEM). The proposed method in this paper consists of three main steps; 1) generating the normalized DSM for two epochs, 2) performing segmentation and structural classification of image segments in order to generate multi temporal classification maps, 3) producing the change maps. The ability of the proposed algorithm is evaluated in a rapid developing urban area in Tehran, Iran in a 9-years interval. The obtained results represent that the ground and bare soil decrease for about $-1.37 \%$ and low-rise buildings also decrease for about $-9.7 \%$. Moreover, the class of high-rise buildings increases for about $+16.4 \%$ which conforms making new constructions in addition to renovation of low-rise buildings.
\end{abstract}

\section{INTRODUCTION}

Considering urban developments, evaluation of damages to urban buildings due to natural disasters, identification of urban change trends, including the rate of destruction and renovation of buildings and the construction of new buildings are very important matters for urban managers and decision makers.

Several methods have been developed for detecting urban changes using various types of remote sensing data to meet a wide range of implicational needs (Singh, 1998).

Change detection algorithms can be divided into two general two-dimensional and three-dimensional categories by considering the type of data and the method of execution (Reinartz, 2016a).

Many previous studies in urban change detection have been carried out to identify the changes in urban buildings based on remote sensing images, without digital elevation models, which was largely due to no access to high-altitude digital models (Bouziani et al., 2010; Brunner et al., 2010; Huang et al., 2014; Vakalopoulou et al., 2015).

However, problems in the 2D change detection methods, including large amounts of data processing, even for small areas, the inability to perform volumetric calculations (Martha et al., 2010; Tian et al., 2014b; Waser et al., 2008) due to the absence of altitude data and the easier production of threedimensional data in recent years caused researchers to the use of three-dimensional change detection methods (Daniel and Doran, 2013; Gruen, 2013).

Some of the researches have proposed three dimensional change detection algorithms based on multi temporal DSMs differencing (Gong et al., 2000; Heller et al., 2001).
In some researches 3D information extracted from DSMs are added to the 2D change detection results obtained from multi temporal image differencing. However, careful post-processing should also be performed to avoid the extension of $2 \mathrm{D}$ errors into the 3D change map (Champion et al., 2009).

Chaabouni and Chouayakh (2010), proposed the postprocessing steps such as morphological operations and contextual knowledge generation in order to remove virtual changes and to keep only the real changes in the obtained $3 \mathrm{D}$ change detection results.

Since altitude information plays a very important role in building recognition, in this paper a three-dimensional change detection method is proposed using two digital elevation models in a 9-year time series.

\section{PROPOSED METHOD}

In this paper, 3D change detection for built up areas are considered due to construction of new buildings in flat areas and renovation of low-rise buildings and converting them into high-rise ones. As it is illustrated in figure 1, the proposed 3D change detection algorithm in this paper has three main steps; 1) Generating the normalized DSMs for both epochs. By subtracting DSM which contains 3D objects such as buildings and trees from DTM which only contains 2D structures such as bare soil and roads, normalized DSM will be generated which contains all kinds of 3D objects.

2) Performing object based image analysis composed of image segmentation and structural classification on normalized DSM. In this step of the proposed algorithm, the multi-temporal classification maps are obtained for change detection.

\footnotetext{
* Corresponding author
} 
3) Producing change maps based on the multi-temporal classification results of normalized DSMs. The generated change maps contain new constructed buildings and renovated high-rise buildings.

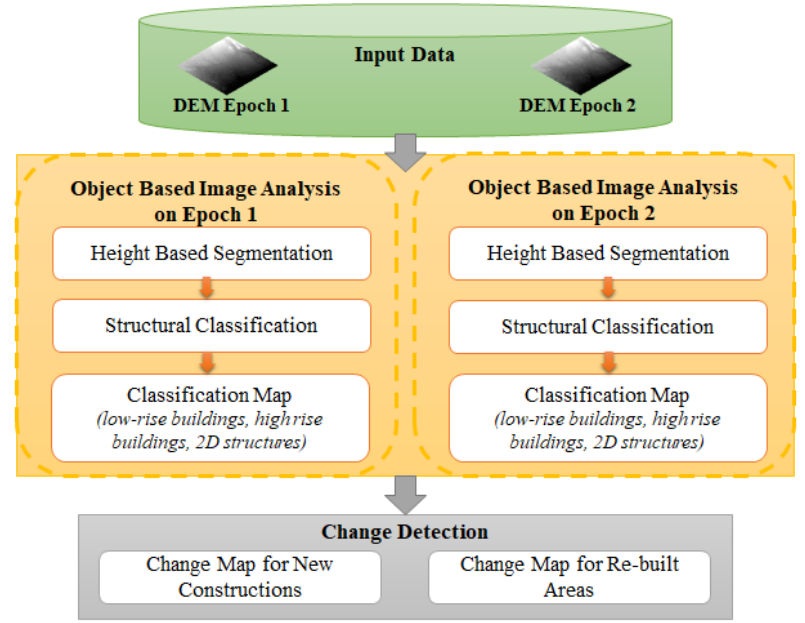

Figure 1. Structure of the proposed 3D change detection method

\subsection{Proposed Object Based Image Analysis}

In the first step of the proposed object based image analysis strategy in this paper, the multi-resolution segmentation technique is applied on the normalized DSM content in order to segment it into regions. The multi-resolution segmentation procedure starts with single image objects of one pixel and repeatedly merges a pair of image objects into larger ones. The merging decision is based on the local homogeneity criterion, describing the similarity between adjacent image objects. This homogeneity criterion is modified by compactness and shape parameters. Moreover, a scale parameter is defined for modifying the size of the segmented regions. The procedure stops when there are no more possible merges (Tabib mahmoudi, et al., 2013).

In the second step, a knowledge based classification process is performed on segmented regions. Therefore, it is necessary to gather proper knowledge about the segmented regions. As the objective of this research is performing three dimensional changes detection in urban areas based on digital surface models, ground (bare soil and roads), low-rise buildings and high-rise buildings are the pre-defined object classes. Therefore, height based thresholds in addition to some of the structural features such as area, rectangular-fit and elongation those are suitable for building recognition provides a rich knowledge base.

After generating the above mentioned structural features for each of the segmented regions, and by considering the height thresholds, proper reasoning rules are performed for generating the classification map containing ground, low-rise and high-rise building classes.

After generating the classification maps for two epochs, by subtracting them from each other, three dimensional changes detection can determine the percentages of constructing new buildings, renovation of low-rise buildings into high-rise ones and destruction of the buildings in order to increase ground and streets.

\section{EXPERIMNTAL RESULTS}

The potential of the proposed $3 \mathrm{D}$ change detection method in this paper is evaluated based on the digital elevation models generated from Tehran, Iran in a 9-years interval. Digital surface models are generated based on topographic maps and digital train models are generated based on two dimensional layers of the maps. Figure 2 illustrates the generated normalized DSM of the same region on the years 2001 and 2010.
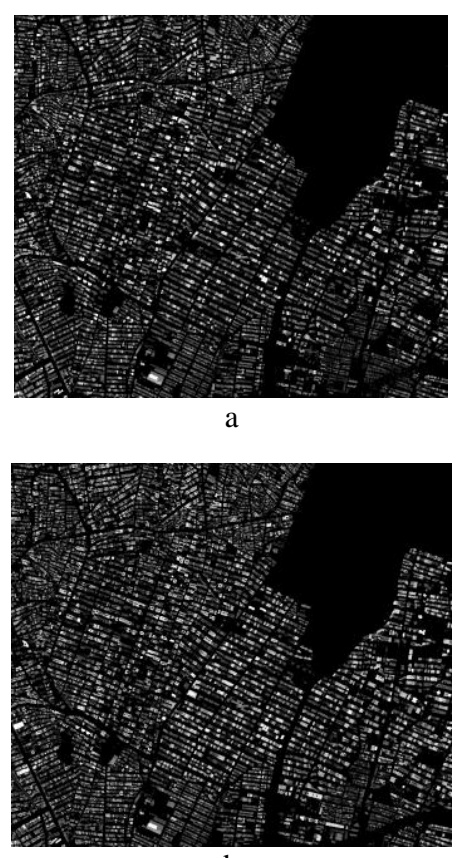

b

Figure 2. Normalized DSM of the year a) 2001, b) 2010

Performing object based image analysis on the normalized DSMs generates classification maps containing high-rise buildings, low-rise buildings and two dimensional structures (bare soil and roads). Figure 3 illustrates the classification maps for both epochs.
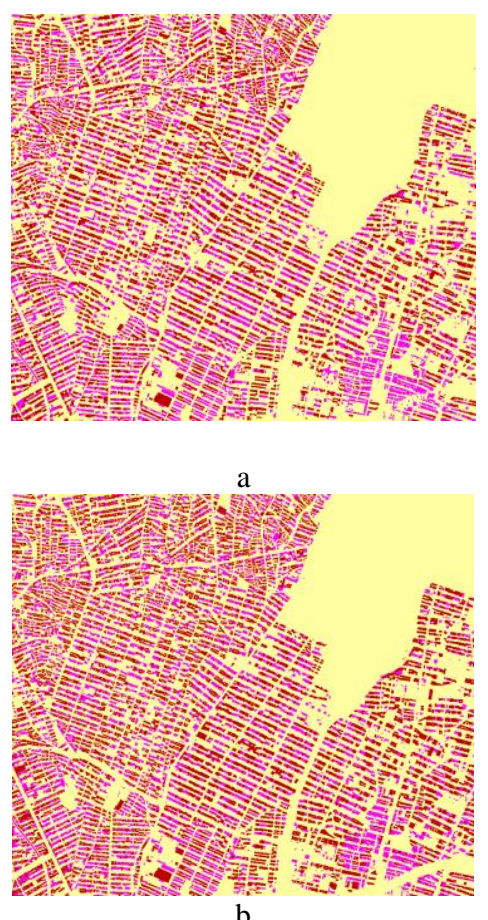

Building (Low-Rise)

Building (High-Rise)

Ground

Figure 3. Classification maps for both epochs a) 2001, b) 2010 
The obtained results from change detection between height and structural based classification maps are illustrated in figure 4.

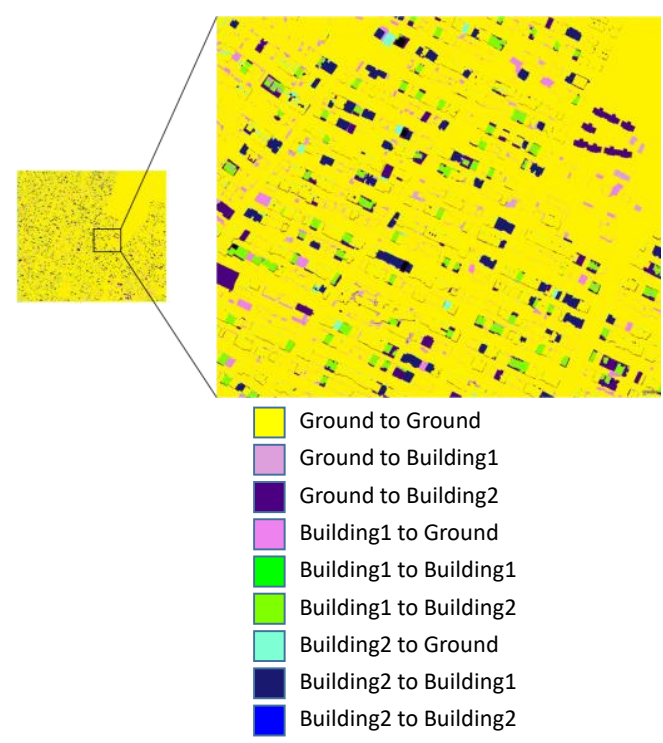

Figure 4. The generated change map

The changes from ground class to the low-rise and high-rise building classes represent that $7.03 \%$ new constructions are added to this study area in a 9-years interval. On the other hand, urban sprawl caused some changes in the elevation of the existing buildings. These changes converted $18.98 \%$ of the lowrise buildings into the high-rise ones.

Table 1 depicts some statistical details containing initial and last areas of each object class and the difference percentage determined from multi-temporal changes.

Table 1. Change area statistical information

\begin{tabular}{|c|c|c|c|c|}
\hline $\begin{array}{c}\text { Object } \\
\text { classes }\end{array}$ & $\begin{array}{c}\text { Area } \\
\text { Epoch 1) }\end{array}$ & $\begin{array}{c}\text { Area } \\
\text { (Epoch 2) }\end{array}$ & $\begin{array}{c}\text { Differencing } \\
\text { Area }\end{array}$ & $\begin{array}{c}\text { Difference } \\
\text { Percentage }\end{array}$ \\
\hline Ground & 7544059 & 7440924 & -103135 & $-1.36 \%$ \\
\hline $\begin{array}{c}\text { Low-rise } \\
\text { building }\end{array}$ & 1767309 & 1595641 & -171668 & $-9.714 \%$ \\
\hline $\begin{array}{c}\text { High-rise } \\
\text { building }\end{array}$ & 1675916 & 1950719 & 274803 & $16.397 \%$ \\
\hline
\end{tabular}

Figure 5 compares the performed changes percentages in ground, low-rise and high-rise buildings in a 9-years interval.

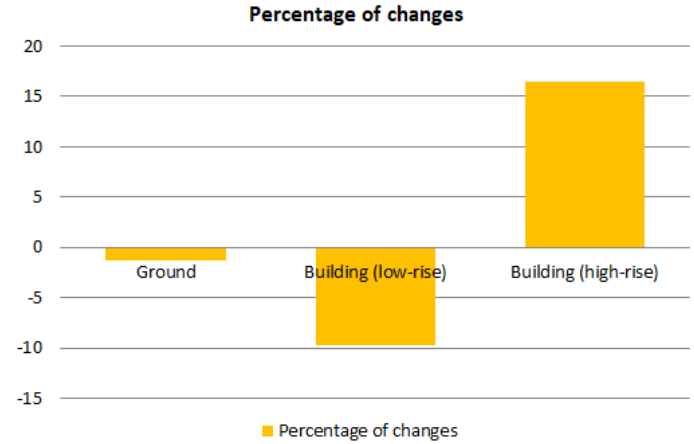

Figure 5. Percentages of changes in ground, low-rise and high-rise buildings

\section{CONCLUSION}

Identification of urban change trends, including the rate of destruction and renovation of buildings and the construction of new buildings are considered in this research. The proposed 3D change detection algorithm is based on digital elevation models in the forms of normalized DSM. By performing object based image analysis composed of image segmentation and structural classification on digital elevation models, classification maps are generated for change detection. Three dimensional change detection results in this paper represent some changes between ground object class and low-rise and high-rise buildings in a developing urban area in Tehran, Iran.

The obtained results from performing 3D change detection show that constructing new buildings led to $1.36 \%$ decrease in the ground area. On the other hand, renovation and reconstruction of low-rise buildings led to $9.714 \%$ decrease in area of low-rise buildings. Developing nature of the study area increases the area of the high-rise buildings which is about $16.4 \%$.

Therefore, the obtained change detection results in the selected urban area confirm new constructions in bare soil and changing low-rise buildings into high-rise ones due to urban vertical developments.

\section{REFERENCES}

Bouziani, M., Goïta, K., He, D.-C., 2010. Automatic change detection of buildings in urban environment from very high spatial resolution images using existing geodatabase and prior knowledge. ISPRS J. Photogram. Rem. Sens. 65 (1), 143- 153.

Brunner, D., Lemoine, G., Bruzzone, L., 2010. Earthquake damage assessment of buildings using VHR optical and SAR imagery. Geosci. Rem. Sens., IEEE Trans. on 48 (5), 2403 2420.

Chaabouni-Chouayakh, H., Krauss, T., d'Angelo, P., Reinartz, P., 2010. 3D change detection inside urban areas using different digital surface models. Int. Arch. Photogram., Rem. Sens. Spatial Inform. Sci. 38, 86-91.

Champion, N., Rottensteiner, F., Matikainen, L., Liang, X., Hyyppä, J., Olsen, B., 2009. A test of automatic building change detection approaches. Int. Arch. Photogram., Rem. Sens. Spatial. Inform. Sci. 38 (Part 3/W4), 145-150.

Daniel, S., Doran, M.-A., 2013. GeoSmartCity: geomatics contribution to the smart city. In: Proceedings of the 14th Annual International Conference on Digital Government Research, pp. 65-71.

Gong, P., Biging, Greg.S., Standiford, R., 2000. Technical note: use of digital surface model for hardwood rangeland monitoring. J. Range Manage. 53 (6), 622-626.

Gruen, A., 2013. Next generation smart cities-the role of geomatics. BBC 26.17: 32.81 G 547 (25), 25.

Heller, A.J., Leclerc, Y.G., Luong, Q.-T., 2001. Framework for robust 3D change detection. In: International Symposium on Remote Sensing, pp. 639-649.

Huang, X., Zhang, L., Zhu, T., 2014. Building change detection from multitemporal high-resolution remotely sensed images based on a morphological building index. Select. Topics Appl. Earth Observ. Rem. Sens., IEEE J. 7 (1), 105-115. 
Martha, T.R., Kerle, N., Jetten, V., van Westen, C.J., Kumar, K.V., 2010. Landslide volumetric analysis using Cartosat-1derived DEMs. IEEE Geosci. Rem. Sens. Lett. 7 (3), 582-586.

Reinartz, Rongjun Qin, Jiaojiao Tian, 2016a. 3D change detection - Approaches and applications, ISPRS Journal of Photogrammetry and Remote Sensing 122 (2016) 41-56.

Singh, A., 1989. Digital Change Detection Techniques Using Remotely-Sensed Data. Int. J. Rem. Sens. 10 (6), 989-1003.

Tian, J., Nielsen, A., Reinartz, P., 2014b. Improving change detection in forest areas based on stereo panchromatic imagery using kernel MNF. IEEE Trans. Geosci. Rem. Sens. 52 (11), $7130-7139$

Tabib Mahmoudi, F., Samadzadegan, F., Reinartz, P., 2013. Object oriented image analysis based on multi-agent recognition system. Computers \& Geosciences 54 (2013), 219-230.

Vakalopoulou, M., Karantzalos, K., Komodakis, N., Paragios, N., 2015. Simultaneous registration and change detection in multitemporal, very high resolution remote sensing data. In: Proceedings of the IEEE Conference on Computer Vision and Pattern Recognition Workshops, pp. 61-69.

Waser, L., Baltsavias, E., Ecker, K., Eisenbeiss, H., FeldmeyerChriste, E., Ginzler, C., Küchler, M., Zhang, L., 2008. Assessing changes of forest area and shrub encroachment in a mire ecosystem using digital surface models and CIR aerial images. Rem. Sens. Environ. 112 (5), 1956-1968. 